Journal of Applied Analysis

Vol. 5, No. 1 (1999), pp. 95-112

\title{
ON A TYPE OF HYPERBOLIC VARIATIONAL-HEMIVARIATIONAL INEQUALITIES
}

\author{
P.D. PANAGIOTOPOULOS and G. POP \\ Received May 5, 1998 and, in revised form, November 18, 1998
}

\begin{abstract}
We consider a hyperbolic variational-hemivariational initial value problem on a vector valued functions space. Using a regularization procedure and a Barbu result we obtain an existence result for a problem independent on $u^{\prime}$.
\end{abstract}

\section{Introduction}

The hyperbolic and the parabolic hemivariational (or variational-hemivariational) initial value problem were studied by several authors. Interesting results concerning the existence property for the parabolic case can be found in [8], [9] or in [3]. Existence results for the hyperbolic case have been obtained in [13] and in [7]. The problem studied in this paper differs by the problems considered in [13] or in [7] due to the presence of the subgradient of a convex function $\psi$ and due to the absence of the terms which contain $u^{\prime}$.

We will use a regularization procedure which combines the Yoshida approximation with the regularization technique based on mollifiers (see, e.g.

1991 Mathematics Subject Classification. 34G20, 35L.

Key words and phrases. Hyperbolic problem, generalized gradient of Clarke.

ISSN 1425-6908 (c) Heldermann Verlag. 
[2]). The existence property for the regularized problem is a consequence of a Barbu result.

In the last part of this paper we give applications concerning beams and plates adhesively connected with a support.

Throughout the paper we assume the following hypotheses:

$\left(\mathbf{H}_{1}\right)$ The separable real Hilbert space $V$ is compactly and densely imbedded in $H=L^{2}\left(\Omega ; \mathbb{R}^{N}\right)$, where $\Omega$ is an open, bounded subset of $\mathbb{R}^{m}$. One identifies $H$ with its dual space $H^{*}$, thus one has $V \subset H=H^{*} \subset V^{*}$.

$\left(\mathbf{H}_{2}\right) A \in \mathcal{L}\left(V, V^{*}\right)$ is a self-adjoint, coercive operator (i.e. there is a positive constant $\omega$ such that $(A u, u) \geq \omega\|u\|_{V}^{2}$, for every $u \in V$.)

$\left(\mathbf{H}_{3}\right) \psi: V \rightarrow \mathbb{R}$ is a lower semicontinuous, convex function such that $\partial \psi$ is bounded (i.e. it maps bounded subsets in bounded subsets).

$\left(\mathbf{H}_{4}\right) j: \mathbb{R}^{N} \rightarrow \mathbb{R}$ is a Lipschitz-continuous function.

$\left(\mathbf{H}_{5}\right) u_{0} \in V, u_{1} \in H$ and $f \in L^{2}(0, T ; H)$.

$\left(\mathbf{H}_{6}\right)$ If $\left(u_{n}\right)_{n}$ is bounded in $L^{\infty}(0, T ; V), u_{n} \rightarrow u$ in $L^{1}(0, T ; H)$ and $\left(v_{n}^{*}\right)_{n}$ is a sequence such that $v_{n}^{*}(t) \in \partial \psi\left(u_{n}(t)\right)$ a.e. on $(0, T)$, for every $n$, then there is a subsequence of $\left(v_{n}^{*}\right)_{n}$, which is weakly-* convergent in $L^{\infty}\left(0, T ; V^{*}\right)$ to $v^{*}$ and $v^{*}(t) \in \partial \psi(u(t))$ a.e. on $(0, T)$.

We denote by $\left(\right.$, ) the duality between $V$ and $V^{*}$ and by $(,)_{H}$ the inner product of $H$. \|\|$_{V}$ is the $V$-norm, \|\|$_{H}$ is the $H$-norm and || is the euclidean norm in $\mathbb{R}^{N}$. As a consequence of $\left(\mathbf{H}_{\mathbf{1}}\right)$, one has

$$
(u, v)=(u, v)_{H} \text { for every } u \in H \text { and } v \in V .
$$

The aim of this article is to give an existence result for the following problem:

Problem (P). Find $u \in L^{\infty}(0, T ; V)$, with $u^{\prime} \in L^{\infty}(0, T ; H), u^{\prime \prime} \in$ $L^{1}\left(0, T ; V^{*}\right)$, and find $\chi \in L^{\infty}(0, T ; H)$ such that

$$
\left\{\begin{array}{l}
u^{\prime \prime}(t)+A u(t)+\partial \psi(u(t))+\chi(t) \ni f(t), \text { a.e. on }(0, T), \\
\chi(t) \in U^{*}\left(\partial_{c} j(U u(t))\right), \text { a.e. on }(0, T), \\
u(0)=u_{0}, u^{\prime}(0)=u_{1},
\end{array}\right.
$$

where $\partial_{c}$ denotes the generalized gradient introduced by Clarke (see [4]).

Here, and in the sequel, $U: H \rightarrow L^{2}\left(\Omega^{\prime} ; \mathbb{R}^{N}\right)$ is defined by $U v=\left.v\right|_{\Omega^{\prime}}$, where $\Omega^{\prime}$ is an open subset of $\Omega$ and $U^{*}$ denotes the adjoint of $U$, i.e.

$$
U^{*}: L^{2}\left(\Omega^{\prime} ; \mathbb{R}^{N}\right) \rightarrow H,\left(U^{*} u\right)(x)= \begin{cases}v(x) & \text { if } x \in \Omega^{\prime} \\ 0 & \text { otherwise. }\end{cases}
$$

The derivatives which appear in this paper are derivatives in the sense of distributions on $(0, T)$. 
Remark 1. Assume, generally, that $(X, \mathcal{B}, \mu)$ is a positive measure space. If $C$ is a measurable multivalued operator defined on $\mathbb{R}^{N}$, we can define the extension of $C$ to $L^{2}\left(X ; \mathbb{R}^{N}\right), \bar{C}$ by setting:

$$
f \in \bar{C}(u) \Leftrightarrow f(x) \in C(u(x)) \quad \mu \text {-a.e. on } X \text {. }
$$

In this paper, we use the same notation for $C$ and its extension, $\bar{C}$.

Remark 2. If $u$ is a solution of the problem $(\mathbf{P})$, then $u$ satisfies:

$$
\begin{aligned}
& \left(u^{\prime \prime}(t), v\right)+(A u(t), v)+\psi(v+u(t))-\psi(u(t))+\int_{\Omega^{\prime}} j^{0}(u(x, t) ; v(x)) d x \\
\geq & \int_{\Omega} f(x, t) \cdot v(x) d x \forall v \in V, \text { a.e. } t \in(0, T) .
\end{aligned}
$$

Remark 3. The idea of using the operator $U$ in the formulation of $(\mathbf{P})$ came from [10].

As a first step in proving an existence result for problem $(\mathbf{P})$, we will consider a regularized problem and we will give an existence result for it.

Let $\rho \in C_{0}^{\infty}\left(\mathbb{R}^{N} ; \mathbb{R}\right)$ be a nonnegative function such that

$$
\int_{\mathbb{R}^{N}} \rho(x) d x=1 \text { and for } x \text { with }|x| \geq 1, \rho(x)=0 .
$$

For every positive integer $n$, we consider $\rho_{n} \in C_{0}^{\infty}\left(\mathbb{R}^{N}, \mathbb{R}\right), \rho_{n}(x)=n^{N} \rho(n x)$ and $j_{n}: \mathbb{R}^{N} \rightarrow \mathbb{R}, j_{n}=j * \rho_{n}$, where $*$ denotes the convolution product.

Lemma 1. If $j$ satisfies $\left(\mathbf{H}_{\mathbf{4}}\right)$, then

1. For every positive integer $n$, we have: $j_{n} \in C^{\infty}\left(\mathbb{R}^{N} ; \mathbb{R}\right)$ and $j_{n}, j_{n}^{\prime}$ are Lipschitz-continuous functions. In addition, there is a constant $L$, such that

$$
\left|j_{n}^{\prime}(x)\right| \leq L, \text { for } x \in \mathbb{R}^{N} \text { and for every } n .
$$

2. If $\left(x_{n}\right)_{n}$ converges in $\mathbb{R}^{N}$ to $x$, then

$$
\limsup _{n} j_{n}^{\prime}\left(x_{n}\right) \cdot y \leq j^{0}(x ; y), \text { for } y \in \mathbb{R}^{N} .
$$

Here $j^{0}(;)$ is the directional derivative of Clarke for $j$, i.e.

$$
j^{0}(x ; y)=\limsup _{\substack{z \rightarrow x \\ t \rightarrow 0^{+}}} \frac{j(z+t y)-j(z)}{t} .
$$


Proof. 1. follows immediately from the formula which defines $j_{n}$.

2. follows from [14], Lemma 1 or from [6], Lemma 2.1.

Let $\phi: H \rightarrow \mathbb{R} \cup\{\infty\}, \phi(u)= \begin{cases}\psi(u)+\frac{1}{4}(A u, u) & \text { if } u \in V \\ \infty & \text { otherwise. }\end{cases}$

Due to the hypotheses $\left(\mathbf{H}_{\mathbf{2}}\right)$ and $\left(\mathbf{H}_{\mathbf{3}}\right)$, it follows that $\phi$ is a convex, proper and lower semicontinuous function on $H$. For each positive integer $n$, one defines:

$$
\phi_{n}: H \rightarrow \mathbb{R}, \phi_{n}(u)=\inf _{v \in H}\left\{\frac{n}{2}\|u-v\|_{H}^{2}+\phi(v)\right\} .
$$

Remark that $\phi_{n}$ is Fréchet-differentiable and its derivative, $\phi_{n}^{\prime}$, is Lipschitzcontinuous. (See [1], Corollary 2.2, Chap. 2.)

Let $\left(f_{n}\right)_{n}$ be a sequence in $H^{1}(0, T ; H)$, which converges in $L^{2}(0, T ; H)$ to $f$.

From $\left(\mathbf{H}_{\mathbf{1}}\right)$, one follows that $\{u \in V: A u \in H\}$ is a dense subset of $V$. Let $u_{0 n} \in V$, be a sequence such that $A u_{0 n} \in H$, for every $n$ and $u_{0 n} \stackrel{V}{\rightarrow} u_{0}$. As $V$ is densely imbedded in $H$, there is a sequence $\left(u_{1 n}\right)_{n} \in V$ such that $u_{1 n} \stackrel{H}{\rightarrow} u_{1}$.

For every positive integer $n$, we consider the following regularized problem:

Problem $\left(\mathbf{P}_{\mathbf{n}}\right)$. Find $u \in L^{\infty}(0, T ; V)$, with $u^{\prime} \in L^{\infty}(0, T ; H), u^{\prime \prime} \in$ $L^{1}\left(0, T ; V^{*}\right)$ such that

$$
\left\{\begin{array}{lr}
u^{\prime \prime}(t)+\frac{1}{2} A u(t)+\phi_{n}^{\prime}(u(t))+\left(U^{*} \circ j_{n}^{\prime} \circ U\right) & (u(t))=f_{n}(t), \\
u(0)=u_{0 n}, u^{\prime}(0)=u_{1 n} & \text { a.e. on }(0, T),
\end{array}\right.
$$

Theorem 1. There exists a sequence $\left(u_{n}\right)_{n} \subset C(0, T ; H) \cap L^{\infty}(0, T ; V)$ such that for every $n$ we have: $u_{n}^{\prime} \in L^{\infty}(0, T ; V) \cap C\left(0, T ; V^{*}\right), u_{n}^{\prime \prime} \in$ $L^{\infty}(0, T ; H), A u_{n} \in L^{\infty}(0, T ; H)$ and

$$
\begin{cases}u_{n}^{\prime \prime}(t)+\frac{1}{2} A u_{n}(t)+\phi_{n}^{\prime}\left(u_{n}(t)\right)+\left(U^{*} \circ j_{n}^{\prime} \circ U\right) & \left(u_{n}(t)\right)=f_{n}(t), \\ u_{n}(0)=u_{0 n}, u_{n}^{\prime}(0)=u_{1 n} . & \text { a.e. on }(0, T),\end{cases}
$$

Proof. For every $n, u_{n}$ is the solution of $\left(\mathbf{P}_{\mathbf{n}}\right)$. The existence property for $\left(\mathbf{P}_{\mathbf{n}}\right)$ and the required properties for $u_{n}$ follow from [1], Theorem 1.5, Chap. 5. 
Theorem 2. The following properties hold:

(i) The sequence $\left(u_{n}^{\prime}\right)_{n}$ is bounded in $L^{\infty}(0, T ; H)$;

(ii) The sequence $\left(u_{n}\right)_{n}$ is bounded in $L^{\infty}(0, T ; V)$;

(iii) The sequence $\left(\phi_{n}\left(u_{n}\right)\right)_{n}$ is bounded from above in $L^{\infty}(0, T)$;

(iv) The sequence $\left(J_{n}\left(u_{n}\right)\right)_{n}$ is bounded in $L^{\infty}(0, T ; V)$;

(v) The sequence $\left(\phi_{n}^{\prime}\left(u_{n}\right)\right)_{n}$ is bounded in $L^{\infty}\left(0, T ; V^{*}\right)$;

(vi) The sequence $\left(A u_{n}\right)_{n}$ is bounded in $L^{\infty}\left(0, T ; V^{*}\right)$;

(vii) The sequence $\left(\left(U^{*} \circ j_{n}^{\prime} \circ U\right)\left(u_{n}\right)\right)_{n}$ is bounded in $L^{\infty}(0, T ; H)$;

(viii) The sequence $\left(u_{n}^{\prime \prime}\right)_{n}$ is bounded in $L^{1}\left(0, T ; V^{*}\right)$;

(ix) The sequence $\left(\frac{d}{d t}\left(J_{n} \circ u_{n}\right)\right)_{n}$ is bounded in $L^{\infty}(0, T ; H)$;

where $J_{n}=\left(I+\frac{1}{n} \partial \phi\right)^{-1}$.

Proof. Let $t$ be fixed in $[0, T]$. Taking the inner product in the equation from (1) with $u_{n}^{\prime}(s)$, then integrating $s$ between 0 and $t$, one obtains:

$$
\begin{aligned}
& \int_{0}^{t}\left(f_{n}(s), u_{n}^{\prime}(s)\right)_{H} d s=\int_{0}^{t}\left(u_{n}^{\prime \prime}(s), u_{n}^{\prime}(s)\right)_{H} d s \\
& +\frac{1}{2} \int_{0}^{t}\left(A u_{n}(s), u_{n}^{\prime}(s)\right) d s+\int_{0}^{t}\left(\phi_{n}^{\prime}\left(u_{n}(s)\right), u_{n}^{\prime}(s)\right)_{H} d s \\
& +\int_{0}^{t}\left(\left(U^{*} \circ j_{n}^{\prime} \circ U\right)\left(u_{n}(s)\right), u_{n}^{\prime}(s)\right)_{H} d s .
\end{aligned}
$$

On the other hand,

$$
\begin{aligned}
\int_{0}^{t}\left(u_{n}^{\prime \prime}(s), u_{n}^{\prime}(s)\right)_{H} d s & =\frac{1}{2} \int_{0}^{t} \frac{d}{d s}\left\|u_{n}^{\prime}(s)\right\|_{H}^{2} d s \\
& =\frac{1}{2}\left\|u_{n}^{\prime}(t)\right\|_{H}^{2}-\frac{1}{2}\left\|u_{n}^{\prime}(0)\right\|_{H}^{2} \\
& =\frac{1}{2}\left\|u_{n}^{\prime}(t)\right\|_{H}^{2}-\frac{1}{2}\left\|u_{1 n}\right\|_{H}^{2} \geq \frac{1}{2}\left\|u_{n}^{\prime}(t)\right\|_{H}^{2}-c,
\end{aligned}
$$




$$
\begin{aligned}
\frac{1}{2} \int_{0}^{t}\left(A u_{n}(s), u_{n}^{\prime}(s)\right) d s & =\frac{1}{4} \int_{0}^{t} \frac{d}{d s}\left(A u_{n}(s), u_{n}(s)\right) d s \\
& =\frac{1}{4}\left(A u_{n}(t), u_{n}(t)\right)-\frac{1}{4}\left(A u_{0 n}, u_{0 n}\right) \\
& \geq \frac{1}{4} \omega\left\|u_{n}(t)\right\|_{V}^{2}-c \\
\int_{0}^{t}\left(\left(U^{*} \circ j_{n}^{\prime} \circ U\right)\left(u_{n}(s)\right), u_{n}^{\prime}(s)\right)_{H} d s & \\
\geq & \int_{0}^{t}\left\|\left(U^{*} \circ j_{n}^{\prime} \circ U\right)\left(u_{n}(s)\right)\right\|_{H}\left\|u_{n}^{\prime}(s)\right\|_{H} d s \\
& \geq-L \int_{0}^{t}\left\|u_{n}^{\prime}(s)\right\|_{H} d s \geq-\frac{L}{2}\left(T+\int_{0}^{t}\left\|u_{n}^{\prime}(s)\right\|_{H}^{2} d s\right)
\end{aligned}
$$

and

$$
\begin{aligned}
\int_{0}^{t}\left(f_{n}(s), u_{n}^{\prime}(s)\right)_{H} d s & \leq \frac{1}{2}\left\|f_{n}\right\|_{L^{2}(0, T ; H)}^{2}+\frac{1}{2} \int_{0}^{t}\left\|u_{n}^{\prime}(s)\right\|_{H}^{2} d s \\
& \leq c+\frac{1}{2} \int_{0}^{t}\left\|u_{n}^{\prime}(s)\right\|_{H}^{2} d s .
\end{aligned}
$$

Let us estimate the term $\int_{0}^{t}\left(\phi_{n}^{\prime}\left(u_{n}(s)\right), u_{n}^{\prime}(s)\right)_{H} d s$. Let $\psi_{1}: V \rightarrow \mathbb{R}, \psi_{1}(u)=$ $\psi(u)+(1 / 4)(A u, u)$.

As $\psi_{1}$ is a convex, coercive, lower semicontinuous function on $V$, the range of $\partial \psi_{1}$ is $V^{*}$. Thus, there is a $v_{0} \in V$ such that $0_{V^{*}} \in \partial \psi_{1}\left(v_{0}\right)$, i.e. $\psi_{1}(u) \geq \psi_{1}\left(v_{0}\right)$, for every $u \in V$. Then, $\phi(u) \geq \phi\left(v_{0}\right)$, for every $u \in H$.

Let $n$ be fixed. From the definition of $\phi_{n}$, one results

$$
\phi_{n}\left(v_{0}\right) \leq \phi_{n}(u) \leq \phi(u)=\psi_{1}(u) \text {, for every } u \in V .
$$

If $v_{n}^{*} \in \partial \psi_{1}\left(u_{0 n}\right)$, one obtains

$$
\psi_{1}\left(v_{0}\right)-\phi_{n}\left(u_{0 n}\right) \geq \psi_{1}\left(v_{0}\right)-\psi_{1}\left(u_{0 n}\right) \geq\left(v_{n}^{*}, v_{0}-u_{0 n}\right),
$$

(we used the fact that the sequence $\left(u_{0 n}\right)_{n}$ is included in $V$.) According to $\left(\mathbf{H}_{3}\right), \partial \psi$ is a bounded operator on $V$. The sequence $\left(u_{0 n}\right)_{n}$ is convergent in $V$, thus it is bounded in $V$. For every $u \in V, \partial \psi_{1}(u)=\partial \psi(u)+(1 / 2) A u$. 
It follows that $\left(v_{n}^{*}\right)_{n}$ is bounded in $V^{*}$. From (7), one obtains:

$$
\psi_{1}\left(v_{0}\right)-\phi_{n}\left(u_{0 n}\right) \geq-c, \text { for every } n .
$$

Therefore, for every $n$,

$$
\begin{aligned}
\int_{0}^{t}\left(\phi_{n}^{\prime}\left(u_{n}(s)\right), u_{n}^{\prime}(s)\right)_{H} d s & =\int_{0}^{t} \frac{d}{d s} \phi_{n}\left(u_{n}(s)\right) d s \\
& =\phi_{n}\left(u_{n}(t)\right)-\phi_{n}\left(u_{0 n}\right) \geq \phi_{n}\left(v_{0}\right)-\psi_{1}\left(v_{0}\right)-c .
\end{aligned}
$$

On the other hand, $\psi_{1}\left(v_{0}\right)=\phi\left(v_{0}\right)=\lim _{n} \phi_{n}\left(v_{0}\right)$. Thus,

$$
\int_{0}^{t}\left(\phi_{n}^{\prime}\left(u_{n}(s)\right), u_{n}^{\prime}(s)\right)_{H} d s \geq-c_{1} .
$$

From (2), (3), (4), (5), (6) and (10), one gets

$$
\frac{1}{2}\left\|u_{n}^{\prime}(t)\right\|_{H}^{2}+\frac{1}{4} \omega\left\|u_{n}(t)\right\|_{V}^{2} \leq \frac{L+1}{2} \int_{0}^{t}\left\|u_{n}^{\prime}(s)\right\|_{H}^{2} d s+c,
$$

for every $n$ and for all $t \in[0, T]$. But, $u_{n}$ is an absolutely continuous function from $[0, T]$ to $V$, and $u_{n}^{\prime}$ is an absolutely continuous function from $[0, T]$ to $H$. Using Gronwall's inequality, from (11) we obtain (i) and (ii).

From (2), (3), (4), (5), (6), (8), (9) and (i), (ii) one deduces

$$
\begin{aligned}
\phi_{n}\left(u_{n}(t)\right) & =\phi_{n}\left(u_{0 n}\right)+\int_{0}^{t}\left(\phi_{n}^{\prime}\left(u_{n}(s)\right), u_{n}^{\prime}(s)\right)_{H} d s \\
& =\phi_{n}\left(u_{0 n}\right)+\int_{0}^{t}\left(f_{n}(s), u_{n}^{\prime}(s)\right)_{H}-\int_{0}^{t}\left(u_{n}^{\prime \prime}(s), u_{n}^{\prime}(s)\right)_{H} d s \\
& -\int_{0}^{t}\left(A u_{n}(s), u_{n}^{\prime}(s)\right) d s-\int_{0}^{t}\left(\left(U^{*} \circ j_{n}^{\prime} \circ U\right)\left(u_{n}(s)\right), u_{n}^{\prime}(s)\right)_{H} d s \\
& \leq \psi_{1}\left(v_{0}\right)+c_{1},
\end{aligned}
$$

for every $t \in[0, T]$ and every $n$. Therefore, (iii) is satisfied.

As $J_{n}$ are non-expansive functions from $H$ to $H$, it consequently follows that, if $v_{n}(t)=J_{n}\left(u_{n}(t)\right)$, then $\left\|v_{n}^{\prime}(t)\right\|_{H} \leq\left\|u_{n}^{\prime}(t)\right\|_{H}$ a.e. $t \in(0, T)$ and (ix) is implied by (i).

For every $n$ and for every $t \in[0, T]$, we have $v_{n}(t) \in \mathcal{D}(\partial \phi) \subset \mathcal{D}(\phi)=V$, thus

$$
\psi_{1}\left(v_{n}(t)\right)=\phi\left(v_{n}(t)\right)=\phi\left(J_{n}\left(u_{n}(t)\right)\right) \leq \phi_{n}\left(u_{n}(t)\right) \leq \psi_{1}\left(v_{0}\right)+c_{1} .
$$


(See [1], Theorem 2.2, Chap. 2.)

As $\psi_{1}$ is coercive on $V$, for every positive number $c$, there is a positive number $R$ such that

$$
\psi_{1}(v) \leq c \Rightarrow\|v\|_{V} \leq R
$$

Consequently, (iv) is satisfied.

For $u \in V$, we have $\partial \phi(u) \subset \partial \psi_{1}(u)$. One obtains

$$
\phi_{n}^{\prime}\left(u_{n}(t)\right) \in \partial \phi\left(v_{n}(t)\right) \subset \partial \psi_{1}\left(v_{n}(t)\right), \forall t \in[0, T], \forall n .
$$

From (iv) and $\left(\mathbf{H}_{\mathbf{3}}\right)$, it follows (v). From $\left(\mathbf{H}_{\mathbf{2}}\right)$ and (ii), it follows (vi). Lemma 1,1) proves (vii) and (1) together with the previous assertions prove (viii).

Lemma 2. Let $X, Y$ two reflexive spaces such that $X$ is compactly imbedded in $Y$. Let $\left(u_{n}\right)_{n}$ be a bounded sequence in $L^{\infty}(0, T ; X)$ such that $\left(u_{n}^{\prime}\right)_{n}$ is weakly convergent in $L^{1}(0, T ; Y)$. Then, there are an $u \in L^{\infty}(0, T ; Y)$ and a subsequence $\left(u_{n_{k}}\right)_{k}$ such that

$$
\begin{aligned}
u_{n_{k}}(t) & \rightarrow u(t) \text { in } Y, \text { a.e. on }(0, T) \text { and } \\
u_{n_{k}} & \rightarrow u \text { in } L^{p}(0, T ; Y), \forall p \in(1, \infty) .
\end{aligned}
$$

Proof. From the hypotheses of this lemma, one follows that the sequence $\left(u_{n}\right)_{n}$ is bounded in $L^{2}(0, T ; Y)$. Passing to a subsequence, we can assume that $\left(u_{n}\right)_{n}$ is weakly-convergent in $L^{2}(0, T ; Y)$. Let $u$ be the weak-limit of $\left(u_{n}\right)_{n}$ in $L^{2}(0, T ; Y)$. Firstly, we will prove that

$$
u_{n}(t) \rightarrow u(t) \text { weakly in } Y \text {, a.e. on }(0, T) .
$$

As $u_{n}$ and $u_{n}^{\prime}$ are in $L^{1}(0, T ; Y)$, it follows that $u_{n}$ can be identified with an absolutely continuous function from $[0, T]$ to $Y$. For any $n$, for any $v \in Y^{*}$ and for any $t \in[0, T]$,

$$
\begin{aligned}
u_{n}(t) & =u_{n}(0)+\int_{0}^{t} u_{n}^{\prime}(s) d s \\
\left(u_{n}(t), v\right)_{Y, Y^{*}} & =\left(u_{n}(0), v\right)_{Y, Y^{*}}+\int_{0}^{t}\left(u_{n}^{\prime}(s), v\right)_{Y, Y^{*}} d s \\
& =\left(u_{n}(0), v\right)_{Y, Y^{*}}+\left\langle u_{n}^{\prime}, \chi_{(0, t)} \otimes v\right\rangle_{L^{1}(0, T ; Y) ; L^{\infty}\left(0, T ; Y^{*}\right)}
\end{aligned}
$$

where $\chi_{(0, t)}$ is the characteristic function of the set $(0, t)$. (For $\theta:(0, T) \rightarrow \mathbb{R}$ and $v \in Y^{*}, \theta \otimes v:(0, T) \rightarrow Y^{*}$ is defined by $(\theta \otimes v)(t)=\theta(t) v$. $)$

As $\left(u_{n}(0)\right)_{n}$ is a bounded sequence in $X$, which is compactly imbedded in $Y$, one can assume that $\left(u_{n}(0)\right)_{n}$ is convergent in $Y$. The sequences 
from the right side of the previous equality being convergent, there is an $\bar{u}:[0, T] \rightarrow Y$ such that

$$
\left(u_{n}(t), v\right)_{Y, Y^{*}} \rightarrow(\bar{u}(t), v)_{Y, Y^{*}}, \forall t \in[0, T], \forall v \in Y^{*} .
$$

We are going to prove that $\bar{u}=u$. It is obvious that $\bar{u} \in L^{\infty}(0, T ; Y)$. Let $\theta$ be in $L^{1}\left(0, T ; Y^{*}\right)$. Then, a.e. on $(0, T), \forall n$,

$$
\left|\left(u_{n}(t), \theta(t)\right)_{Y, Y^{*}}\right| \leq c\left\|u_{n}(t)\right\|_{X}\|\theta(t)\|_{Y^{*}} \leq c_{1}\|\theta(t)\|_{Y^{*}} .
$$

Due to Lebesgue's dominated convergence theorem, from (12) and (13) one obtains

$$
\begin{aligned}
& \left\langle u_{n}, \theta\right\rangle_{L^{\infty}(0, T ; Y), L^{1}\left(0, T ; Y^{*}\right)} \\
= & \int_{0}^{T}\left(u_{n}(s), \theta(s)\right)_{Y, Y^{*}} d s \rightarrow \int_{0}^{T}(\bar{u}(s), \theta(s))_{Y, Y^{*}} d s=\langle\bar{u}, \theta\rangle_{L^{\infty}(0, T ; Y), L^{1}\left(0, T ; Y^{*}\right)} .
\end{aligned}
$$

Therefore, $\left(u_{n}\right)_{n}$ converges to $\bar{u}$ weakly-* in $L^{\infty}(0, T ; Y)$. One follows that $\left(u_{n}\right)_{n}$ converges to $\bar{u}$ weakly in $L^{2}(0, T ; Y)$. Consequently, $u=\bar{u}$.

Let us prove that $\left(u_{n}\right)_{n}$ converges in $L^{p}(0, T ; Y)$ to $u$, for $p \in(1, \infty)$. Due to the assumed boundedness of $\left(u_{n}\right)_{n}$, it is sufficient to prove that $\left(u_{n}(t)\right)_{n}$ converges in $Y$ to $u(t)$, a.e. on $(0, T)$. The sequence $\left(u_{n}(t)\right)_{n}$ is bounded in $X$ a.e. on $(0, T)$, therefore it is a relatively compact subset of $Y$. On the other side, $u_{n}(t) \rightarrow u(t)$ weakly in $Y$ a.e. on $(0, T)$. One obtains that $\left(u_{n}(t)\right)_{n}$ converges in $Y$ to $u(t)$ a.e. on $(0, T)$.

Theorem 3. The problem $(\mathbf{P})$ has a solution.

Proof. Let $\left(u_{n}\right)_{n}$ be a sequence as in Theorem 1. Passing to a subsequence (if necessary), from Theorem 2 (i), (ii), (iv), (ix), hypothesis $\left(\mathbf{H}_{\mathbf{5}}\right)$ and Lemma 2 , it follows that there are $u$ and $v$ in $L^{2}(0, T ; H)$, such that

$$
u_{n} \rightarrow u, v_{n} \rightarrow v \text { in } L^{2}(0, T ; H),
$$

where $v_{n}(t)=J_{n}\left(u_{n}(t)\right)$ for $t \in(0, T)$. The proof of our assertion has several steps.

Step 1. $u_{n} \rightarrow u, v_{n} \rightarrow v$ weakly-* in $L^{\infty}(0, T ; V)$.

Proof. According to Theorem 2 (ii), (iv) $\left(u_{n}\right)_{n}$ and $\left(v_{n}\right)_{n}$ are bounded sequences in $L^{\infty}(0, T ; V)$, the latter being continuously imbedded in $L^{2}(0, T ; H)$. Passing to a subsequence, we can assume that there are $\bar{u}$ and $\bar{v}$ in $L^{\infty}(0, T ; V)$, such that

$$
u_{n} \rightarrow \bar{u}, v_{n} \rightarrow \bar{v}, \text { weakly-* in } L^{\infty}(0, T ; V) .
$$

It follows that $\left(u_{n}\right)_{n}$ and $\left(v_{n}\right)_{n}$ are weakly convergent in $L^{2}(0, T ; H)$, to $\bar{u}$, respectively to $\bar{v}$. From (14), one obtains the assertion stated in Step 1. 
Step 2. $u=v$.

Proof. For every $n, J_{n}=(I+(1 / n) \partial \phi)^{-1}$. Consequently, for every $n$ and a.e. on $(0, T)$, we have

$$
u_{n}(s) \in J_{n}\left(u_{n}(s)\right)+\frac{1}{n} \partial \phi\left(J_{n}\left(u_{n}(s)\right)\right) .
$$

But, $\partial \phi\left(J_{n}\left(u_{n}(s)\right)\right) \subset \partial \psi_{1}\left(J_{n}\left(u_{n}(s)\right)\right)$. Due to $\left(\mathbf{H}_{\mathbf{3}}\right)$ and by Theorem 2, (iv), there is a constant $c$ such that

$$
\partial \psi_{1}\left(J_{n}\left(u_{n}(s)\right)\right) \subset\left\{v^{*} \in V^{*}:\left\|v^{*}\right\|_{V^{*}}<c\right\}, \forall n \text {, a.e. on }(0, T) \text {. }
$$

Thus

$$
\left\|u_{n}(s)-J_{n}\left(u_{n}(s)\right)\right\|_{V^{*}} \leq \frac{1}{n} c, \forall n, \text { a.e. on }(0, T) .
$$

On the other side, as a consequence of (14), we can assume that

$u_{n}(s)-J_{n}\left(u_{n}(s)\right) \rightarrow u(s)-v(s)$ in $H$ and, consequently, in $V^{*}$ a.e. on $(0, T)$. It follows from (15) that $u=v$.

Step 3. $\left(A u_{n}\right)_{n}$ and $\left(A v_{n}\right)_{n}$ converge to $A u$ weakly-* in $L^{\infty}\left(0, T ; V^{*}\right)$. Proof. As $A$ is a self-adjoint operator from $V$ to $V^{*}$, the assertions derive easily from Step 1.

Step 4. $\quad \phi_{n}^{\prime}\left(u_{n}\right) \rightarrow v^{*}+(1 / 2) A u$ weakly-* in $L^{\infty}\left(0, T ; V^{*}\right)$, with $v^{*}(s) \in$ $\partial \psi(u(s))$, a.e. on $(0, T)$.

Proof. The sequence $\left(\phi_{n}^{\prime}\left(u_{n}\right)\right)_{n}$ is bounded in $L^{\infty}\left(0, T ; V^{*}\right)$ (see Theorem 2, $(\mathrm{v}))$. For every $n$, a.e. on $(0, T)$,

$$
\phi_{n}^{\prime}\left(u_{n}(t)\right) \in \partial \phi\left(v_{n}(t)\right) \subset \partial \psi_{1}\left(v_{n}(t)\right)=\partial \psi\left(v_{n}(t)\right)+\frac{1}{2} A v_{n}(t) .
$$

Conform Lemma $2, v_{n} \rightarrow u$ in $L^{2}(0, T ; H)$. One can apply $\left(\mathbf{H}_{\mathbf{6}}\right)$ for $v_{n}^{*}=$ $\phi_{n}^{\prime}\left(u_{n}\right)-(1 / 2) A v_{n}$. Then, one can assume that there is a $v^{*}$ in $L^{\infty}\left(0, T ; V^{*}\right)$ such that

$$
\begin{aligned}
-\frac{1}{2} A v_{n}+\phi_{n}^{\prime}\left(u_{n}\right) & \rightarrow v^{*} \text { weakly-* in } L^{\infty}\left(0, T ; V^{*}\right), \\
v^{*}(t) & \in \partial \psi(u(t)), \text { a.e. on }(0, T) .
\end{aligned}
$$

Step 5. $\left(U^{*} \circ j_{n}^{\prime} \circ U\right)\left(u_{n}\right) \rightarrow \chi$ weakly-* in $L^{\infty}(0, T ; H)$ and $\chi \in$ $L^{\infty}(0, T ; H)$ satisfies

$$
\chi(t) \in U^{*}\left(\partial_{c} j(U u(x, t))\right) \text { a.e. on }(0, T) .
$$

Proof. The existence of $\chi$ is a consequence of Theorem 2 (vii). Let $Q_{T}=$ $\Omega^{\prime} \times(0, T)$ and let $\widetilde{Q_{T}}=\left(\Omega \backslash \Omega^{\prime}\right) \times(0, T)$. Due to the separability of $\mathbb{R}^{N}$ and 
using the upper-semicontinuity of $j^{0}$, in order to have (16) it is sufficient to prove that for every positive function $\theta \in L^{\infty}\left(Q_{T}\right)$ and every $\xi \in \mathbb{R}^{N}$,

$$
\int_{Q_{T}} \chi(x, t) \cdot \xi \theta(x, t) d x d t \leq \int_{Q_{T}} j^{0}(u(x, t) ; \xi) \theta(x, t) d x d t
$$

and that for every function $\theta \in L^{\infty}\left(\widetilde{Q_{T}}\right)$ and every $\xi \in \mathbb{R}^{N}$,

$$
\int_{Q_{T}} \chi(x, t) \cdot \xi \theta(x, t) d x d t=0 .
$$

Let us prove (17). Let $\theta$ and $\xi$ be as before; taking into account that $\chi$ is the weak-* limit of $\left(\left(U^{*} \circ j_{n}^{\prime} \circ U\right)\left(u_{n}\right)\right)_{n}$ in $L^{\infty}(0, T ; H)$, one can write:

$$
\begin{aligned}
\int_{Q_{T}} \chi(x, t) \cdot \xi \theta(x, t) d x d t & =\int_{0}^{T}\left(\chi(t), U^{*}(\xi \theta(t))\right)_{H} d t \\
& =\lim _{n} \int_{0}^{T}\left(\left(U^{*} \circ j_{n}^{\prime} \circ U\right)\left(u_{n}(t)\right), U^{*}(\xi \theta(t))\right)_{H} d t \\
& =\lim _{n} \int_{Q_{T}} j_{n}^{\prime}\left(u_{n}(x, t)\right) \cdot \xi \theta(x, t) d x d t .
\end{aligned}
$$

As $u_{n} \rightarrow u$ in $L^{2}\left(0, T ; L^{2}\left(\Omega^{\prime} ; \mathbb{R}^{N}\right)\right)$, which can be identified with $L^{2}\left(Q_{T} ; \mathbb{R}^{N}\right)$ we can assume that

$$
u_{n}(x, t) \rightarrow u(x, t) \text { a.e. on } \Omega^{\prime} \times(0, T) .
$$

Due to Lemma 1, one obtains

$$
\begin{aligned}
\limsup _{n} j_{n}^{\prime}\left(u_{n}(x, t)\right) \cdot \xi & \leq j^{0}(u(x, t)) \cdot \xi, \\
\left|j_{n}^{\prime}\left(u_{n}(x, t)\right)\right| & \leq L,
\end{aligned}
$$

a.e. on $\Omega^{\prime} \times(0, T)$. Thus,

$$
\begin{aligned}
& \underset{n}{\limsup } \int_{Q_{T}} j_{n}^{\prime}\left(u_{n}(x, t)\right) \cdot \xi \theta(x, t) d x d t \\
\leq & \int_{Q_{T}} \limsup _{n} j_{n}^{\prime}\left(u_{n}(x, t)\right) \cdot \xi \theta(x, t) d x d t \leq \int_{Q_{T}} j^{0}(u(x, t)) \cdot \xi \theta(x, t) d x d t .
\end{aligned}
$$

The assertion follows from (19) and (20). As $\left(U^{*} \circ j_{n}^{\prime} \circ U\right)\left(u_{n}\right)=0$ a.e. on $\widetilde{Q_{T}}$, analogously, we derive (18).

Step 6. $u_{n}^{\prime} \rightarrow u^{\prime}$ weakly-* in $L^{\infty}(0, T ; H)$. 
Proof. $\left(u_{n}^{\prime}\right)_{n}$ is a bounded sequence in $L^{\infty}(0, T ; H)$. We can assume that $u_{n}^{\prime} \rightarrow z$ weakly-* in $L^{\infty}(0, T ; H)$. In order to prove that $u^{\prime}=z$, we will show that

$$
\int_{0}^{T} u(t) \theta^{\prime}(t) d t=-\int_{0}^{T} z(t) \theta(t) d t, \forall \theta \in C_{0}^{\infty}(0, T),
$$

or, equivalently,

$$
\int_{0}^{T}(u(t), w)_{H} \theta^{\prime}(t) d t=-\int_{0}^{T}(z(t), w)_{H} \theta(t) d t, \forall \theta \in C_{0}^{\infty}(0, T), \forall w \in H .
$$

Let $\theta$ and $w$ be as before. One has

$$
\begin{aligned}
\int_{0}^{T}(u(t), w)_{H} \theta^{\prime}(t) d t & =\lim _{n} \int_{0}^{T}\left(u_{n}(t), w\right)_{H} \theta^{\prime}(t) d t, \\
-\int_{0}^{T}(z(t), w)_{H} \theta(t) d t & =-\lim _{n} \int_{0}^{T}\left(u_{n}^{\prime}(t), w\right)_{H} \theta(t) d t .
\end{aligned}
$$

But the terms from the right-hand side of the previous equalities are equal.

Step 7. $u_{n}^{\prime \prime} \rightarrow u^{\prime \prime}$ weakly in $L^{1}\left(0, T ; V^{*}\right)$.

Proof. As $f_{n} \rightarrow f$ in $L^{1}\left(0, T ; V^{*}\right)$, due to (1), from the previous steps it follows that $\left(u_{n}^{\prime \prime}\right)_{n}$ is weakly convergent in $L^{1}\left(0, T ; V^{*}\right)$. Conform Step 6, $u_{n}^{\prime} \rightarrow u^{\prime}$ weakly-* in $L^{\infty}(0, T ; H)$ and, consequently, weakly in $L^{1}\left(0, T ; V^{*}\right)$.

The assertion follows using similar arguments with those used in the proof of the previous step.

Step 8. $u^{\prime}(0)=u_{1}, u(0)=u_{0}$.

Proof. Let $n$ be fixed. For all $t \in[0, T]$,

$$
u_{n}(t)=u_{0 n}+\int_{0}^{t} u_{n}^{\prime}(s) d s
$$

For $v \in H$, conform Step 6 and Lemma 2, the following hold:

$$
\begin{aligned}
\left(\int_{0}^{t} u_{n}^{\prime}(s) d s, v\right)_{H} & =\int_{0}^{T}\left(u_{n}^{\prime}(s), v \chi_{(0, t)}(s)\right)_{H} d s \rightarrow\left(\int_{0}^{t} u^{\prime}(s) d s, v\right)_{H}, \\
\left(u_{n}(t), v\right)_{H} & \rightarrow(u(t), v)_{H},\left(u_{0 n}, v\right)_{H} \rightarrow\left(u_{0}, v\right)_{H} .
\end{aligned}
$$


Passing to weak-limit by $n$ in (21), we obtain that

$$
u(t)=u_{0}+\int_{0}^{t} u^{\prime}(s) d s .
$$

But $u \in L^{\infty}(0, T ; H)$ and $u^{\prime} \in L^{\infty}(0, T ; H)$; thus,

$$
u(t)=u(0)+\int_{0}^{t} u^{\prime}(s) d s .
$$

It follows that $u(0)=u_{0}$. By a similar argument for $\left(u_{n}^{\prime}\right)_{n}$ and the space $V^{*}$, one obtains that $u^{\prime}(0)=u_{1}$.

Step 9. $u^{\prime \prime}(t)+A u(t)+v^{*}(t)+\chi(t)=f(t)$ a.e. on $(0, T)$. Proof. As $u^{\prime \prime}+A u+v^{*}+\chi-f \in L^{1}\left(0, T ; V^{*}\right)$, in order to prove the required equality, we will prove that for every $v \in V$ and every $\theta \in L^{\infty}(0, T)$,

$$
\int_{0}^{T}\left(u^{\prime \prime}(t)+A u(t)+v^{*}(t)+\chi(t)-f(t), v\right) \theta(t) d t=0 .
$$

Then, due to the separability of $V$, the assertion in Step 9 will follow immediately.

Let $v \in V$ and $\theta \in L^{\infty}(0, T)$ be as before. From (1) one obtains:

$\int_{0}^{T}\left(u_{n}^{\prime \prime}(t)+\frac{1}{2} A u_{n}(t)+\phi_{n}^{\prime}\left(u_{n}(t)\right)+\left(U^{*} \circ j_{n}^{\prime} \circ U\right)\left(u_{n}(t)\right)-f_{n}(t), v\right) \theta(t) d t=0$.

Passing to limit by $n$ and taking into account that the application $t \mapsto v \theta(t)$ is in the space $L^{1}(0, T ; V)$, one obtains $(22)$.

Proposition 1. Let $V$ be a normed space continuously imbedded in the separable Hilbert space $H$. If $M: H \rightarrow \mathcal{P}(H)$ is a bounded, maximal monotone operator, then $M$ satisfies $\left(\mathbf{H}_{\mathbf{6}}\right)$.

Proof. Let $\left(u_{n}\right)_{n}$ be a bounded sequence in $L^{\infty}(0, T ; V)$, such that $u_{n} \rightarrow u$ in $L^{1}(0, T ; H)$. Let $\left(v_{n}^{*}\right)_{n}$ be such that $v_{n}^{*}(t) \in M u_{n}(t)$ a.e. on $(0, T)$ and for every $n$. As $\mathcal{R}(M) \subset H$, one obtains that $\left(v_{n}^{*}\right)_{n}$ is a bounded sequence in $L^{\infty}(0, T ; H)$. Let us assume that $v_{n}^{*} \rightarrow v^{*}$ weakly-* in $L^{\infty}(0, T ; H)$. We are going to prove that $v^{*}(t) \in M u(t)$ a.e. on $(0, T)$. Let $\left[w, w^{*}\right] \in M$ and $\theta$ a positive function in $L^{\infty}(0, T)$. Then

$$
\int_{0}^{T}\left(w^{*}-v_{n}^{*}(t), w-u_{n}(t)\right)_{H} \theta(t) d t \geq 0, \forall n .
$$


But,

$$
\begin{aligned}
w^{*}-v_{n}^{*} & \rightarrow w^{*}-v^{*} \text { weakly-* in } L^{\infty}(0, T ; H), \\
w-u_{n} & \rightarrow w-u \text { in } L^{1}(0, T ; H) .
\end{aligned}
$$

Passing to limit in (23), one obtains

$$
\int_{0}^{T}\left(w^{*}-v^{*}(t), w-u(t)\right)_{H} \theta(t) d t \geq 0 .
$$

As $\theta$ was chosen arbitrarily among positive functions in $L^{\infty}(0, T)$, we get

$$
\left(w^{*}-v^{*}(t), w-u(t)\right)_{H} \geq 0 \text {, a.e. on }(0, T) \text {. }
$$

On the other hand, $M$ can be identified with a subset of the separable space $H \times H$. Thus, there is a sequence $\left(\left[w_{k}, w_{k}^{*}\right]\right)_{k}$ dense in $M$. For every $k$, one considers the following set:

$$
\mathcal{A}_{k}=\left\{t \in(0, T):(24) \text { is not satisfied for } w=w_{k}, w^{*}=w_{k}^{*}\right\} .
$$

Let $\mathcal{A}$ be $\bigcup_{k} \mathcal{A}_{k}$. Then $\mathcal{A}$ is a set with Lebesgue measure zero.

For $t \in(0, T) \backslash \mathcal{A}$ and for every integer $n$,

$$
\left(w_{n}^{*}-v^{*}(t), w_{n}-u(t)\right)_{H} \geq 0 .
$$

Let $t$ be fixed as before. Let $\left[w, w^{*}\right] \in M$. There is a subsequence $\left(\left[w_{k_{l}}, w_{k_{l}}^{*}\right]\right)_{l}$ such that $w_{k_{l}} \rightarrow w$ in $V$ and $w_{k_{l}}^{*} \rightarrow w^{*}$ in $H$. For every $l,(25)$ is satisfied for $n=k_{l}$. Passing to the limit by $l$, one obtains

$$
\left(w^{*}-v^{*}(t), w-u(t)\right)_{H} \geq 0 .
$$

As $\left[w, w^{*}\right]$ is arbitrarily chosen in $M$, by use of the maximal monotonicity of $M$, one gets that $v^{*}(t) \in M u(t)$.

Remark 4. Let $V$ and $H$, two real Hilbert spaces which satisfy $\left(\mathbf{H}_{\mathbf{1}}\right)$. If $\psi$ satisfies $\left(\mathbf{H}_{3}\right)$ and $\mathcal{R}(\partial \psi) \subset H$, then $\psi$ satisfies $\left(\mathbf{H}_{\mathbf{6}}\right)$.

Proof. Let $\psi_{2}: H \rightarrow \mathbb{R} \cup\{\infty\}, \psi_{2}(u)= \begin{cases}\psi(u)+\|u\|_{H}^{2} & \text { if } u \in V \\ \infty & \text { otherwise. }\end{cases}$

The functional $\psi_{2}$ is proper, convex, lower semicontinuous and coercive on $H$. Consequently, $\partial \psi_{2}$ is a maximal monotone operator. Additionally, we have

$$
\partial \psi_{2}(u)=\partial \psi(u)+2 u, \forall u \in V .
$$

As a consequence of the previous proposition one obtains that $\partial \psi_{2}$ satisfies $\left(\mathbf{H}_{\mathbf{6}}\right)$ and it follows immediately that $\partial \psi$ also satisfies $\left(\mathbf{H}_{\mathbf{6}}\right)$. 


\section{Applications}

2.1. Beam in adhesive contact. We consider an elastic beam obeying linear Hooke's law, which is simply supported at its ends $x=0$ and $x=l$. From its upper side along the segment $\left(l_{1}, l_{2}\right)$ the beam is adhesively connected with a support. Under the beam, at the distance $h$, we consider a deformable support which causes a reaction proportional to its deformation (Winkler support). The displacements of the beam are denoted $u(x, t)$. The action of the adhesive material on the beam is described by a nonmonotone, possibly multivalued, law between $-f_{1}(x, t)$ and $u(x, t)$, where $f_{1}(x, t)$ denotes the reaction force per unit length due to the gluing material. This law may be written in the form (cf. [13], p.51 or [11], p.26)

$$
-f_{1}(x, t) \in \partial_{c} j(u(x, t)) \text { on }\left(l_{1}, l_{2}\right) \times(0, T),
$$

where $j$ is a locally Lipschitz function.

The reaction force due to the Winkler support is

$$
-f_{2}(x, t) \in \beta(u(x, t)) \text { on }(0, l) \times(0, T),
$$

where $\beta(z)=\left\{\begin{array}{ll}k(z-h) & \text { if } z \geq h \\ 0 & \text { if } z<h\end{array}\right.$.

The beam is assumed to have the modulus of elasticity $E$ and the moment of inertia $I$. Then we may write, in the framework of small displacements, the differential equation of the beams

$$
m \frac{\partial^{2} u}{\partial t^{2}}+E I \frac{\partial^{4} u}{\partial x^{4}}=f_{1}(x, t)+f_{2}(x, t)+f_{3}(x, t)
$$

where $f_{3}(x, t)$ is the given loading and $m$ denotes the mass intensity per unit length. We add the boundary conditions

$$
u(t, 0)=u(t, l)=0, \frac{\partial^{2} u}{\partial x^{2}}(t, 0)=\frac{\partial^{2} u}{\partial x^{2}}(t, l)=0
$$

and the initial conditions

$$
u(x, 0)=u_{0}(x), \frac{\partial u}{\partial t}(x, 0)=u_{1}(x) .
$$

We introduce the space $V=H^{2}((0, l)) \cap H_{0}^{1}((0, l))$ which is a Hilbert space for the inner product (cf. [5], p. 220, Remark 4.5)

$$
a(u, v)=\int_{0}^{l} \frac{\partial^{2} u}{\partial x^{2}} \frac{\partial^{2} v}{\partial x^{2}} d x .
$$


Let $\psi_{1}: L^{2}((0, l)) \rightarrow \mathbb{R}, \psi_{1}(v)=\int_{0}^{l}(1 / 2) k(v-h)_{+}^{2}(x) d x$. Then $\psi=\left.\psi_{1}\right|_{V}$ satisfies $\left(\mathbf{H}_{\mathbf{3}}\right)$ and $(27)$ can be written in the form

$$
-f_{2}(t) \in \partial \psi(u(t)) \text { on }(0, T) .
$$

If the operator $A$ is defined by $(A u, v)=(E I / m) a(u, v), \Omega=(0, l)$ and $\Omega^{\prime}=\left(l_{1}, l_{2}\right)$, one obtains that the displacement $u:(0, T) \rightarrow V$ satisfies:

$$
\left\{\begin{array}{l}
u^{\prime \prime}(t)+A u(t)+\partial\left(\frac{1}{m} \psi\right)(u(t))-\frac{1}{m} f_{1}(t) \ni \frac{1}{m} f_{3}(t), \text { a.e. on }(0, T), \\
-f_{1}(t) \in U^{*}\left(\partial_{c} j(U u(t))\right), \text { a.e. on }(0, T), \\
u(0)=u_{0}, u^{\prime}(0)=u_{1},
\end{array}\right.
$$

where $U v=\left.v\right|_{\Omega^{\prime}}$. We remark that $-f_{1}(t) \in U^{*}\left(\partial_{c} j(U u(t))\right)$, a.e. on $(0, T)$ is equivalent with the following two relations:

$$
\begin{aligned}
& -f_{1}(x, t)=0 \text { for } x \notin \Omega^{\prime}, t \in(0, T) \\
& -f_{1}(x, t) \in \partial_{c} j(u(x, t)) \text { for } x \in \Omega^{\prime}, t \in(0, T) .
\end{aligned}
$$

Note that in the case of large displacements the equation (28) becomes (see $[16])$

$$
m \frac{\partial^{2} u}{\partial t^{2}}+E I \frac{\partial^{4} u}{\partial x^{4}}+P \frac{\partial^{2} u}{\partial x^{2}}=f_{1}(x, t)+f_{2}(x, t)+f_{3}(x, t) .
$$

Here $P$ denotes a compressive force $(P>0)$ acting for $x=0$ and $x=l$ along the axis $O x$. Then, in (31) the operator $A$ is defined by

$$
(A u, v)=\frac{E I}{m} a(u, v)+\int_{0}^{l} P \frac{\partial u}{\partial x} \frac{\partial v}{\partial x} d x .
$$

2.2. The case of plates. We assume an analogous problem to the previous one where now $\Omega \subset \mathbb{R}^{2}$ is occupied by a Kirchhoff plate which is simply supported along the boundary $\Gamma$ of $\Omega$. The set $\Omega$ is assumed to be open, bounded with a Lipschitz boundary. We assume that on $\Omega^{\prime} \subset \Omega$ the plate is adhesively supported from its lower side and that the plate is at a distance $h$ under a deformable support. We assume that $\overline{\Omega^{\prime}} \cap \Gamma=\emptyset$. In this case (cf. [5], p. 207) the problem is governed by the relation

$$
\begin{aligned}
& \rho h \frac{\partial^{2} I}{\partial t^{2}}+D \triangle^{2} I=f_{1}(x, t)+f_{2}(x, t)+f_{3}(x, t) \text { on } \Omega \times(0, T) \\
& I=0, M=0 \text { on } \Gamma \\
& I(t=0)=I_{0}, \frac{\partial I}{\partial t}(t=0)=I_{1} \text { in } \Omega
\end{aligned}
$$

where $h$ denotes the thickness of the plate, $\rho$ the density, $I$ the deflection, $M$ the bending moment and $D=E h^{3} /\left[12\left(1-\nu^{2}\right)\right]$ ( $E$ is the modulus of elasticity and $\nu$ is the number of Poisson). For the expression of $M$ as a function 
of $I$ we refer to [5], p. 204 and to [12], p. 216. The forces $f_{1}, f_{2}, f_{3}$ have the same meaning as in the previous example. The bilinear form $a($,$) of$ the plate theory (see [5], p. 210, eq. 4.1) is continuous and coercive on the space $V \times V$, where $V=H^{2}(\Omega) \cap H_{0}^{1}(\Omega)$, thus it defines a inner product on this space and the norm generated by $a$ is equivalent on $V$ with the norm of $H^{2}(\Omega)$ ([5], p. 220, Remark 4.5). The problem considered above can be put in a form analogous to the form (31).

2.3. Beams and plates with fuzzy support conditions. We can use reaction displacement laws with nonfully determined values (fuzzy laws). In order to describe such a law it is necessary to introduce the following nonconvex superpotential (see [15]): Let $I=(a, b)$ and let $I^{\prime}$ be a measurable subset of $I$ such that for every open and nonempty subset $i$ of $I$, meas $\left(i \cap I^{\prime}\right)$ and meas $\left(i \backslash I^{\prime}\right)$ are positive. Let

$$
g: \mathbb{R} \rightarrow \mathbb{R}, \quad g(z)=\left\{\begin{array}{ll}
b_{2} & \text { if } z \in I^{\prime} \\
b_{1} & \text { if } z \notin I^{\prime}
\end{array} \quad \text { with } b_{1}<b_{2}\right.
$$

and $j: \mathbb{R} \rightarrow \mathbb{R}, j(z)=\int_{0}^{z} g(y) d y$. Then

$$
\partial_{c} j(z)= \begin{cases}{\left[b_{1}, b_{2}\right]} & \text { for } z \in \bar{I} \\ \left\{b_{1}\right\} & \text { for } z<a \\ \left\{b_{2}\right\} & \text { for } z>b .\end{cases}
$$

If we want to describe a fuzzy behaviour for a beam which is adhesively connected on $\left(l_{1}, l_{2}\right)$ with a support, then we will write the law $(26)$ in the form (fuzzy law)

$$
-f_{1} \in \partial_{c} j(u)+\partial \phi(u) \text { on }\left(l_{1}, l_{2}\right) \times(0, T),
$$

where $\phi$ is a convex superpotential and $j$ is the nonconvex superpotential introduced above with $b_{1}=-\alpha, b_{2}=\alpha$ and $I=(-\varepsilon, \varepsilon)$. The law (32) describes the fact that for $u \in(-\varepsilon, \varepsilon)$, the reaction force due to the gluing material $-f_{1}$ may takae any value between $-\alpha$ and $\alpha$. (See also [2], pp. 35-36.)

In the case of a plate adhessively connected with a support on $\Omega^{\prime} \subset \Omega$, the law (32) will be replaced by

$$
-f_{1} \in \partial_{c} j(u)+\partial \phi(u) \text { on } \Omega^{\prime} \times(0, T) .
$$




\section{References}

[1] Barbu, V., Nonlinear Semigroups and Differential Equations in Banach Spaces, Noordhoff Int. Publ., Leyden, 1976.

[2] Brézis, H., Analyse Fonctionnelle. Théorie et Applications, Masson, Paris, 1983.

[3] Carl, S., Enclosure of solutions for quasilinear dynamic hemivariational inequalities, Nonlin. World 3 (1996), 281-298.

[4] Clarke, F.H., Optimization and Nonsmooth Analysis, John Wiley, New York, 1983.

[5] Duvaut, G., Lions, J.L., Inequalities in Mechanics and Physics, Springer-Verlag, Berlin, 1976.

[6] Fundo, M., An existence result on a general type of hemivariational inequalities, Appl. Anal. 65 (1997), 373-394.

[7] Goeleven, D., Miettinen, M., Panagiotopoulos, P.D., Dynamic hemivariational inequalities and their applications, (to appear).

[8] Miettinen, M., A parabolic hemivariational inequality, Nonlinear Anal. 26(4) (1996), $725-734$.

[9] Miettinen, M., Panagiotopoulos, P.D., On parabolic hemivariational inequalities and applications, (to appear).

[10] Motreanu, D., Existence of critical points in a general setting, Set-Valued Anal. 3 (1995), 295-305.

[11] Naniewicz, Z., Panagiotopoulos, P.D., Mathematical Theory of Hemivariational Inequalities and Applications, Marcel Dekker, New York, 1995.

[12] Panagiotopoulos, P.D., Inequality Problems in Mechanics and Applications: Convex and Nonconvex Energy Functions, Birkhäuser, Boston, 1985.

[13] Panagiotopoulos, P.D., Hemivariational Inequalities. Applications in Mechanics and Engineering, Springer-Verlag, Berlin, 1993.

[14] Pop, G., Panagiotopoulos, P.D., Naniewicz, Z., Variational- hemivariational inequalities for multidimensional superpotential laws, Int. J. Num. Funct. Anal. Opt. 18 (1997), 827-856.

[15] Rockafellar, R.T., La Théorie des Sous-Gradients et ses Applications à l'Optimization. Fonctions Convexes et Non-Convexes, Les Presses de l'Université de Montréal, Montréal, 1979.

[16] Timoshenko, S., Gere, J.M., Theory of Elastic Stability, 2nd ed., McGraw Hill, New York, 1961.

P.D. Panagiotopoulos

ARISTOTLE UNIVERSITY

Department of Civil EngineERing

54006 Thessaloniki, Greece

AND

Faculty of Mathematics And Physics

RWTH

52056 AaChEN, GERMANy
Gabriela Pop BUCHAREST UNIVERSITY FACUlTy OF MATHEMATICS ACADEMIEI 14, 70109 BuCHAREST ROMAnia 